\title{
Numerical Analysis on the Reaction Zone Structure of a Supersonic Flow CO Chemical Laser*
}

\author{
Wataru MASUDA ${ }^{* *}$ and Hiroyuki HIRAIDE ${ }^{* * *}$
}

\begin{abstract}
The cavity flow field of a supersonic flow $\mathrm{CO}$ chemical laser is simulated numerically and the effects of the nozzle boundary layers, the cavity boundary layers and the mixing process on the laser performance are studied. The thin shear layer approximation is applied to the mixing region and the resulting equations are solved by the CrankNicolson predictor-corrector method. The numerical results show that the development of the mixing layer in the downstream direction is very gradual. The small signal gain coefficient begins to decay at a location where the mixing layer is still a small portion of the total flow width. The predicted distributions of the averaged gain along the flow agree fairly well with the experiment. It is clearly demonstrated that the presence of the boundary layers and the magnitude of the wall catalysis play decisive roles in determining the laser performance.
\end{abstract}

Key Words: Compressible Flow, Reactive Flow, Laser, Chemical Laser, Carbon Monoxide, Numerical Analysis

\section{Introduction}

The CO chemical laser is of considerable interest, since it has great potential as a high energy device and uses less toxic reactants than the HF chemical laser. In the previous study ${ }^{(1)}$, small signal gain coefficients of a supersonic flow $\mathrm{CO}$ chemical laser were measured using a shock tunnel facility. A theoretical model was also developed using a leaky stream tube approach ${ }^{(2)}$ and the numerical results were compared with the measured small signal gain coefficients. It was shown ${ }^{(3)}$, however, that the theoretical model predicted the gain characteristics only qualitatively. The calculations significantly overestimated the small signal gain coefficients. Therefore, in order to develop a more precise theoretical model, the flow field was observed visually $^{(4)}$ utilizing the chemiluminescence from the

* Received 1st March, 1989. Paper No.89-2003 A

* Faculty of Engineering, Nagaoka University of Technology, 1603-1 Kamitomioka, Nagaoka, 940-21, Japan

*** Sony Co. Ltd., 1-7-4 Kohnan, Minato-ku, Tokyo, 108, Japan cavity.

In the previous experiments ${ }^{(1)(4)}$, a high temperature mixture of $\mathrm{CS}_{2}, \mathrm{CS}, \mathrm{S}_{2}, \mathrm{~S}$ and Ar was produced in a shock tube, where the thermal dissociation of $\mathrm{CS}_{2}$ diluted in Ar was accomplished by a reflected shock wave. The shock heated mixture was exhausted through nozzles mounted at the end of the shock tube. Downstream of the nozzle exits, the chemically nonequilibrium flows were mixed with $\mathrm{O}_{2}$ and the chain reaction :

$$
\begin{aligned}
& \mathrm{O}_{2}+\mathrm{S} \Rightarrow \mathrm{SO}+\mathrm{O}, \\
& \mathrm{O}+\mathrm{CS} \Rightarrow \mathrm{CO}+\mathrm{S},
\end{aligned}
$$

produced the vibrationally excited $\mathrm{CO}$ molecules. Three sets of $\mathrm{CS}_{2}$ nozzles and $\mathrm{O}_{2}$ nozzles, as shown in Fig.1, were used in the previous study ${ }^{(1)(4)}$ In Fig. 1, $x$ is the distance from the nozzle exits parallel to the flow and $y$ and $z$ are coordinates perpendicular to the $x$ axis. The $y$ coordinate is parallel to the optical axis. The measurements of small signal gain coefficients were made using four pairs of large circular windows and three pairs of small circular windows, as shown in Fig. 1. A rectangular window was mounted on the lower wall of the cavity. The rectangular window and large circular windows were also used for the visual 
observation of the chemiluminescence ${ }^{(2)}$ from various reactions in the laser cavity.

Figure 2 shows the typical photographs of the How field which were taken in the previous study ${ }^{(4)}$. The photograph obtained from the rectangular window shows that the $\mathrm{CS}_{2}$ and $\mathrm{O}_{2}$ streams collided with each other at the nozzle exit generating oblique shock waves. The photograph also shows clearly that the mixing between the $\mathrm{CS}_{2}$ streams and $\mathrm{O}_{2}$ streams was significantly slow. For such slow mixing, the effects of the nozzle boundary layers cannot be ignored, since the parcels of fluid issuing from the boundary layers occupy a considerably large portion of the mixing layer. In the previous study ${ }^{(4)}$, therefore, the nozzle boundary layers were also estimated for the typical
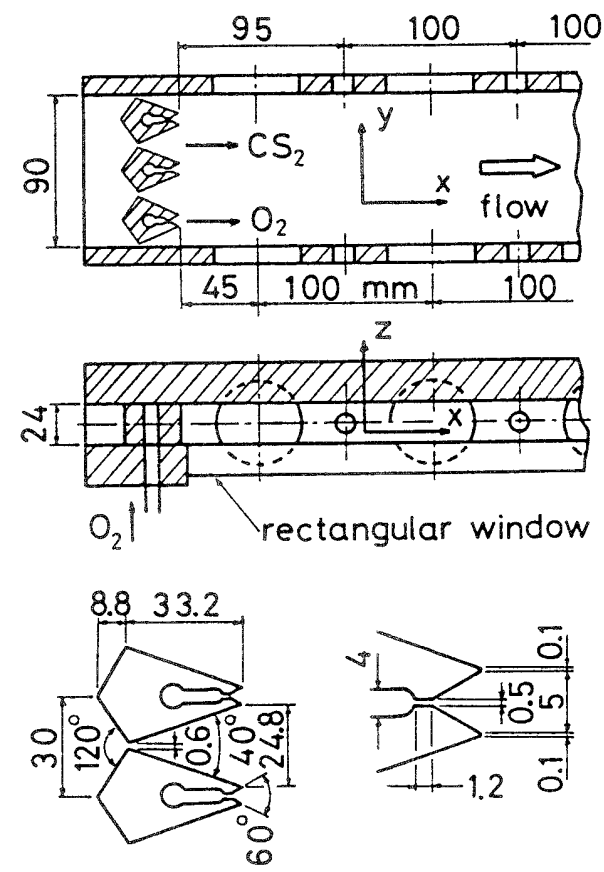

Fig. 1 Schematics of the test section and supersonic nozzles used in the previous experiment
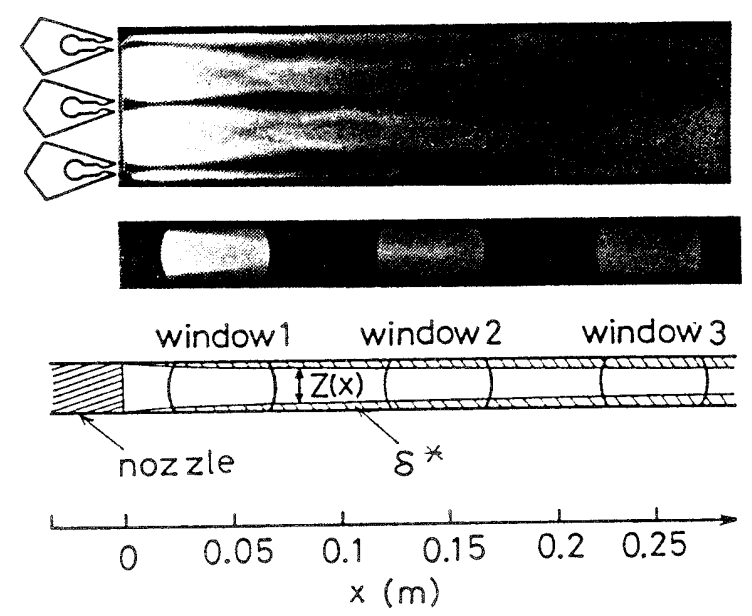

Fig. 2 Chemical luminescence from the cavity experimental condition. The Reynolds numbers of the $\mathrm{CS}_{2}$ nozzle and the $\mathrm{O}_{2}$ nozzle based on the nozzle exit condition and the distances from the throat were $4.2 \times 10^{4}$ and $1.3 \times 10^{4}$, respectively. Therefore, it was assumed that the nozzle boundary layers were laminar. The numerical results showed that the boundary layer thickness $\delta$, the displacement thickness $\delta^{*}$ and the momentum thickness $\theta$ at the exit of the $\mathrm{CS}_{2}$ nozzle were $2.9 \mathrm{~mm}, 1.5 \mathrm{~mm}$ and $0.25 \mathrm{~mm}$, respectively. Therefore, the boundary layers occupied more than $20 \%$ of the flow field at the nozzle exit. It is known that compressibility stabilizes the wake and shear layer. Behrens and $\mathrm{Ko}^{(6)}$ showed experimentally that the turbulent transition of the compressible wake occurred at $x \fallingdotseq 2000 \theta$ at the Mach number of external flow $M_{\infty}=6$. The quasi-one-dimensional calculation $^{(2)}$ showed the frozen Mach number of $\mathrm{CS}_{2}$ nozzle was 7.5. Therefore, the wakes issuing from the nozzles were laminar for a considerably large distance. The photograph obtained from circular windows shows clearly that boundary layers developed along the upper and lower walls of the laser cavity. It is seen that the bright region almost disappeared at the third window.

In the present study, the cavity flow field of the supersonic flow CO chemical laser is simulated numerically and the effects of the nozzle boundary layers, cavity boundary layers and the mixing process on the laser performance are studied. For simplicity, however, the effects of oblique shock waves are neglected in the present study. The nozzle flows and nozzle boundary layers are solved by a method which was developed previously ${ }^{(2)(4)}$. The thin shear layer approximation is applied to the cavity flow field and the resulting equations are solved by the Crank-Nicolson predictor-corrector method". A "quasi-two-dimensional approximation" is used in order to estimate the effects of cavity boundary layers.

\section{Simulation Model and Method}

The flow fields near the upper and lower cavity walls are highly three-dimensional. Therefore, it is not easy to analyse the cavity boundary layers. In the previous study ${ }^{(4)}$, however, the authors assumed that only unmixed $\mathrm{CS}_{2}$ streams issuing from the nozzle existed in the cavity, since they occupied the greater part of the flow. It was also assumed that the cavity boundary layers were laminar, since the Reynolds number based on the edge conditions and $x=0.3 \mathrm{~m}$ was $3.1 \times 10^{5}$. The displacement thickness of the twodimensional compressible boundary layers along flat plates was calculated using the following equation ${ }^{(8)}$ $\delta^{*} / x=\operatorname{Re}_{x}{ }^{-1 / 2}\left[1.73 T_{w} / T_{0}+0.332(\gamma-1) M_{\infty}{ }^{2}\right]$, 
where $\operatorname{Re}_{x}$ is the Reynolds number based on the edge conditions and $x, T_{0}$ is the plenum temperature, $T_{w}$ is the wall temperature and $\gamma$ is the specific heat ratio. In the previous study ${ }^{(4)}, \delta^{*}$ was calculated assuming $T_{w}=300 \mathrm{~K}$ and the edge conditions were equal to the exit conditions of $\mathrm{CS}_{2}$ nozzle. The calculated $\delta^{*}$ is shown in Fig. 2. Since the boundary layer thickness observed from the photograph is several times as large as the calculated displacement thickness, the calculation seems fairly reasonable. The existence of boundary layers causes the convergence of the effective cross sectional area of the cavity. In the present study, let us consider a flow channel with slowly decreasing height $Z(x)$ as shown in Fig. 2. We assume that the flow in the slowly converging channel is "quasi-two-dimensional", that is, all fluid properties are independent of $z$. Using this approximation, we cannot inquire into the flow structure in the boundary layers. However, it is possible to analyse the flow field outside of the boundary layers easily. Figure 2 shows that the bright region is still preserved considerably even at the location of the second circular window. It is also pointed out that the measurement of the small signal gain coefficients was made in the previous study ${ }^{(1)}$ along the centerline of the cavity. In the $x-y$ plane, we use the thin shear layer approximation. The calculation is performed in the region between the centerlines of the neighboring $\mathrm{CS}_{2}$ and $\mathrm{O}_{2}$ nozzles. The fundamental equations for the quasi-two-dimensional chemically and vibrationally nonequilibrium flow can be written as follows:

$$
\begin{aligned}
& \frac{\partial(\rho u Z)}{\partial x}+\frac{\partial(\rho v Z)}{\partial y}=0, \\
& \rho u \frac{\partial u}{\partial x}+\rho v \frac{\partial u}{\partial y}=-\frac{d P}{d x}+\frac{\partial}{\partial y}\left(\mu \frac{\partial u}{\partial y}\right), \\
& \rho u c_{p} \frac{\partial T}{\partial x}+\rho v c_{p} \frac{\partial T}{\partial y}=u \frac{d P}{\partial x}+\mu\left(\frac{\partial u}{\partial y}\right)^{2} \\
& \quad+\frac{\partial}{\partial y}\left(\lambda \frac{\partial T}{\partial y}\right)+\rho \sum_{i} D_{i} c_{p_{i}} \frac{\partial \chi_{i}}{\partial y} \frac{\partial T}{\partial y} \\
& \quad-\rho \sum_{i j}\left(\alpha_{i} \frac{H_{i}-E_{i j}}{\chi_{i}}+\beta_{i j}\right), \\
& P=\rho \sum_{i} \chi_{i} R_{i} T, \\
& \rho u \frac{\partial \chi_{i}}{\partial x}+\rho v \frac{\partial \chi_{i}}{\partial y}=\frac{\partial}{\partial y}\left(\rho D_{i} \frac{\partial \chi_{i}}{\partial y}\right)+\rho \alpha_{i}, \\
& \rho u \frac{\partial E_{i j}}{\partial x}+\rho v \frac{\partial E_{i j}}{\partial y}=\frac{\partial}{\partial y}\left(\rho D_{i} \frac{\partial E_{i j}}{\partial y}\right)+\rho \beta_{i j},
\end{aligned}
$$

where $u$ and $v$ are, respectively, the velocity components in the $x$ and $y$ directions, $P$ is the pressure, $\rho$ is the density, $T$ is the temperature, $\chi_{i}$ is the mass fraction of species $i, E_{i j}$ is the vibrational energy of the $j$-th mode of species $i$ per unit mass of mixture, $H_{i}$ is the enthalpy of species $i$ per unit mass of mixture, $R_{i}$ is the gas constant of species $i, c_{p_{i}}$ is the frozen specific heat at constant pressure of species $i, \mu$ is the viscosity, $\lambda$ is the thermal conductivity, $D_{i}$ is the diffusivity of species $i, \alpha_{i}$ is the rate of formation of $\chi_{i}$ and $\beta_{i j}$ is the rate of formation of $E_{i j}$. In the above equations, the $\mathrm{CO}$ molecule at each different vibrational level is treated as a different species. The coordinate system can be transformed using the following stream function:

$$
\frac{\partial \psi}{\partial x}=-\frac{\rho v Z}{Q}, \quad \frac{\partial \psi}{\partial y}=\frac{\rho u Z}{Q},
$$

where

$$
Q=Z(0) \int_{0}^{h} \rho(0, y) u(0, y) d y=\text { constant, }
$$

and $h$ is the distance between the centerlines of the neighboring $\mathrm{CS}_{2}$ and $\mathrm{O}_{2}$ nozzles. The equation of continuity (4) can be eliminated using the stream function $\psi$. The fundamental equations $(5),(6),(8)$ and (9) transformed into the $(x, \phi)$ plane are as follows :

$$
\begin{aligned}
& \zeta_{1} \frac{\partial^{2} u}{\partial \psi^{2}}+\frac{\partial \zeta_{1}}{\partial \psi} \frac{\partial u}{\partial \psi}=\left(\frac{Q}{Z}\right)^{2}\left(\frac{\partial u}{\partial x}+\frac{1}{\rho u} \frac{d P}{d x}\right), \\
& \zeta_{2} \frac{\partial^{2} T}{\partial \psi^{2}}+\frac{1}{c_{p}}\left\{\frac{\partial}{\partial \psi}\left(c_{p} \zeta_{2}\right)+\zeta_{3} \sum_{i} c_{p_{i}} \frac{\partial \chi_{i}}{\partial \psi}\right\} \frac{\partial T}{\partial \psi} \\
& \quad=\left(\frac{Q}{Z}\right)^{2}\left\{\frac{\partial T}{\partial x}-\frac{1}{\rho c_{p}} \frac{d P}{d x}\right. \\
& \left.\quad+\frac{1}{c_{p} u} \sum_{i j}\left(\alpha_{i} \frac{H_{i}-E_{i j}}{\chi_{i}}+\beta_{i j}\right)\right\}-\frac{\zeta_{1}}{c_{p}}\left(\frac{\partial u}{\partial \psi}\right)^{2}, \\
& \zeta_{3} \frac{\partial^{2} \chi_{i}}{\partial \psi^{2}}+\frac{\partial \zeta_{3}}{\partial \psi} \frac{\partial \chi_{i}}{\partial \psi}=\left(\frac{Q}{Z}\right)^{2}\left(\frac{\partial \chi_{i}}{\partial x}-\frac{\alpha_{i}}{u}\right), \\
& \zeta_{3} \frac{\partial^{2} E_{i j}}{\partial \psi^{2}}+\frac{\partial \zeta_{3}}{\partial \psi} \frac{\partial E_{i j}}{\partial \psi}=\left(\frac{Q}{Z}\right)^{2}\left(\frac{\partial E_{i j}}{\partial x}-\frac{\beta_{i j}}{u}\right),
\end{aligned}
$$

where

$$
\begin{aligned}
& \zeta_{1}=\rho \mu u, \quad \zeta_{2}=\zeta_{1} / \operatorname{Pr}, \quad \zeta_{3}=\zeta_{2} L e_{i}, \\
& \operatorname{Pr}=c_{p} \mu / \lambda, \quad L e_{i}=\rho c_{p} D_{i} / \lambda .
\end{aligned}
$$

The calculation proceeds from the nozzle exit in a downstream direction. The nozzle exit conditions are obtained by the method which was developed previously $^{(4)}$. The boundary conditions at the nozzle centerlines are as follows:

$$
\begin{aligned}
& \text { at } \psi=0 \text { and } 1(y=0 \text { and } h), \\
& \frac{\partial u}{\partial \psi}=\frac{\partial T}{\partial \psi}=\frac{\partial \chi_{i}}{\partial \psi}=\frac{\partial E_{i j}}{\partial \psi}=0 .
\end{aligned}
$$

The flow field is divided with a grid size $\Delta x$ and $\Delta \psi$ with $x=m \cdot \Delta x$ and $\phi=\eta \cdot \Delta \psi$. It is assumed that flow variables are known at the grid points in the $m$-th column and unknown in the $m+1$-th column. The flow variables in the $m+1$-th column are calculated using the Crank-Nicolson predictor-corrector method ${ }^{(6)}$ and assuming the value of $d P / d x$. The calculation is repeated until the following condition is satisfied in the $m+1-$ th column:

$$
\frac{Q}{Z} \int_{0}^{1} \frac{\partial \psi}{\rho u}=h \text {. }
$$

The detailed descriptions of the chemical and vibrational kinetic models are given in the previous papers. ${ }^{(3)(9)}$ The viscosity $\mu$ is calculated using the Chapman-Enskog theory. It is assumed that the gas is 
a mixture of $\mathrm{Ar}, \mathrm{CS}_{2}$ and $\mathrm{O}_{2}$, since the Lennard-Jones potential of most of the other molecules and atoms is not available. It is also assumed that the Prandtl number $P r$ and the Lewis Semenov number $L e_{i}$ are 0.7 and 1.4 , respectively. ${ }^{(16)}$

\section{Results of Calculation}

The present calculations are performed for the nozzle which is shown in Fig. 1 . The temperature $T_{w}$ of the nozzle walls and cavity walls is assumed to be $300 \mathrm{~K}$. The mixture of $\mathrm{CS}_{2}, \mathrm{CS}, \mathrm{S}_{2}, \mathrm{~S}$ and $\mathrm{Ar}$ is generated from the mixture of $\mathrm{CS}_{2}$ and Ar whose initial mole fractions are 0.1 and 0.9 , respectively. The dissociated mixture is assumed to be in thermochemical equilibrium at the plenum. The plenum temperature $T_{o p}$ and the plenum pressure $P_{o p}$ of the dissociated mixture are $3500 \mathrm{~K}$ and $1 \mathrm{MPa}$, respectively. The plenum temperature of $\mathrm{O}_{2}$ is $300 \mathrm{~K}$. Such conditions are the same as those used in the previous experiment ${ }^{(4)}$. In the previous experiment, the plenum pressure of $\mathrm{O}_{2}$ was 60.3 $\mathrm{kPa}$. At this condition, the calculation of the nozzle flow without the boundary layer correction showed that the nozzle exit pressures agreed with each oth$\mathrm{er}^{(1)(2)}$. However, the exit pressures of the $\mathrm{CS}_{2}$ nozzle and the $\mathrm{O}_{2}$ nozzle do not agree with each other if the boundary layer correction is introduced. The nozzle exit pressures must be matched in order to use the thin shear layer approximation in the cavity flow. The calculation in which the effects of the nozzle boundary layers are included shows that the nozzle exit pressures can be matched with each other irrespective of the magnitude of the wall catalysis if $P_{o s}$ is $61.1 \mathrm{kPa}$. Therefore, $P_{o s}=61.1 \mathrm{kPa}$ is used in the present calcu-

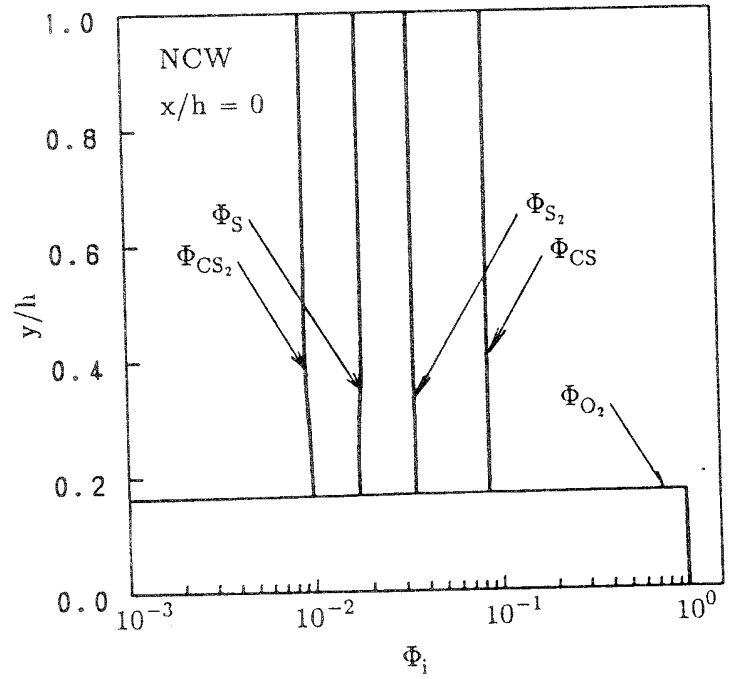

Fig. 3 Distributions of the mole fractions of species at the nozzle exit obtained assuming the noncatalytic wall lations. However, we assume the conditions of the previous experiment and the present calculations are identical, since both conditions are almost equal.

Figures 3 and 4 show the distributions of the mole fractions $\Phi$ of species at the nozzle exit $(x / h=0)$ which are obtained assuming that the nozzle wall is noncatalytic (NCW) and fully catalytic (FCW), respectively. The centerlines of the $\mathrm{O}_{2}$ nozzle and $\mathrm{CS}_{2}$ nozzle are at $y / h=0$ and $y / h=1$, respectively. The nozzle trailing edge is located at $y / h=0.168$. Figure 3 shows that the mass fractions of species at the nozzle wall are almost equal to those at the nozzle centerline if the noncatalytic wall condition is assumed. That means the rate of molecular diffusion in the boundary layer is considerably superior to the rates of chemical reactions. However, as shown in Fig. 4 , the assumption of the fully catalytic wall condition leads to the severe reduction of $\mathrm{CS}$ and $\mathrm{S}$ due to recombination. The CS molecules and $S$ atoms almost disappear at the nozzle wall. The calculations proceed from the nozzle exit toward the downstream direction. In the following calculations, it is assumed in the first place that $Z(x)=$ constant, that is, the cavity boundary layers do not exit. In this case, only the effects of nozzle boundary layers can be examined. Thereafter, the effects of the cavity boundary layer are included in the calculations.

Figures 5 and 6 show the distributions of mole fractions of species at $x / h=20$ which are obtained assuming that the nozzle wall is noncatalytic and fully catalytic, respectively. In these calculations, the effects of cavity boundary layers are not included. Leaving the nozzle trailing edge, CS and S diffuse into

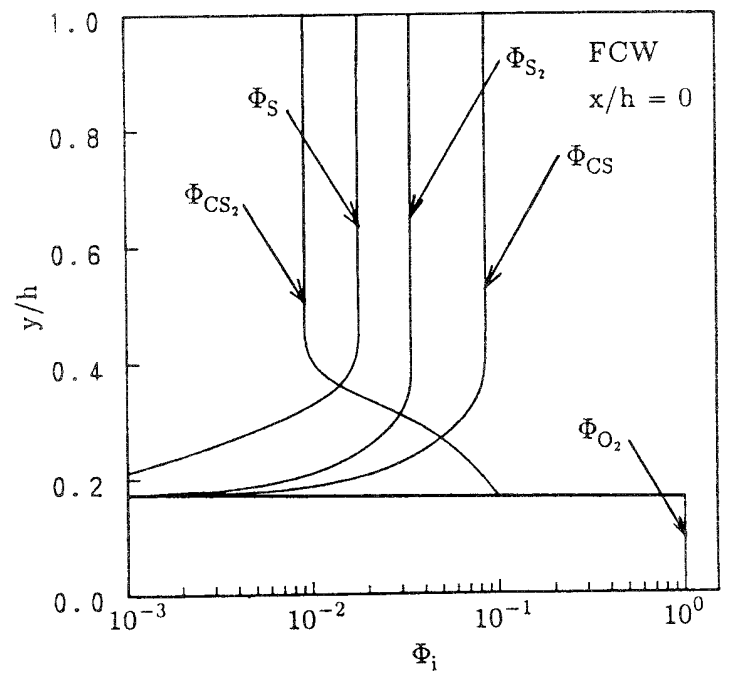

Fig. 4 Distributions of the mole fractions of species at the nozzle exit obtained assuming the fully catalytic wall 
the lower region of the nozzle trailing edge; $\mathrm{O}_{2}$ molecules diffuse into the upper region. Then the $\mathrm{CO}$ molecules are produced in the mixing region by chain reaction (1) and (2). It is clearly seen that the assumption of a noncatalytic nozzle wall conditon leads to the considerably faster formation of $\mathrm{CO}$ than that of fully catalytic nozzle wall condition, since the fuel $\mathrm{CS}$ and the chain carrier $\mathrm{S}$ are scarcely reduced by the noncatalytic wall as shown in Fig. 3.

The distributions of the temperature are shown in Figs. 7 and 8. It is seen from these figures that the temperature distributions in the boundary layer scarcely depend on the magnitude of the wall cataly. sis, since the distributions at the nozzle exit $(x / h=0)$ shown in Figs. 7 and 8 are almost identical. The temperature at the nozzle wall is fixed at $300 \mathrm{~K}$. However,

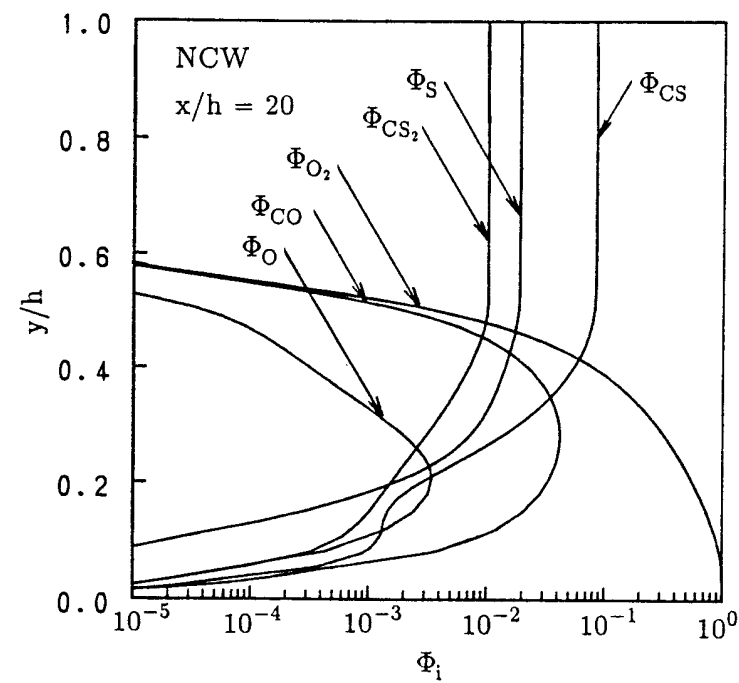

Fig. 5 Distributions of the mole fractions of species at $x / h=20$ obtained assuming the noncatalytic wall

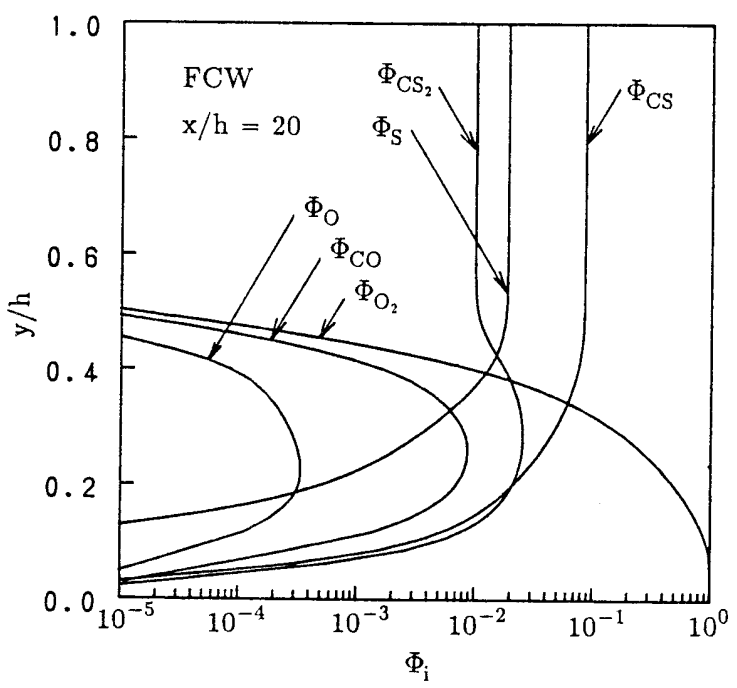

Fig. 6 Distributions of the mole fractions of species at $x / h=20$ obtained assuming the fully catalytic wall in the boundary layer of the $\mathrm{CS}_{2}$ nozzle, the temperature is considerably higher than the wall temperature due to the dissipation. Leaving the nozzle trailing edge, the peak of the temperature is decreased, since the dissipation is decreased due to the reduction in the velocity gradient. Thereafter, the temperature is raised again due to the heat of formation. The assumption of the noncatalytic wall condition results in a higher temperature rise than that of the fully catalytic wall condition, since chemical reactions in the mixing layer are very active.

The velocity distributions are shown in Figs. 9 and 10. It is also seen from the distributions at $x / h=$ 0 of Figs. 9 and 10 that the velocity distributions in the boundary layer scarcely depend on the magnitude of the wall catalysis. In the region $x / h>0$, the velocity in

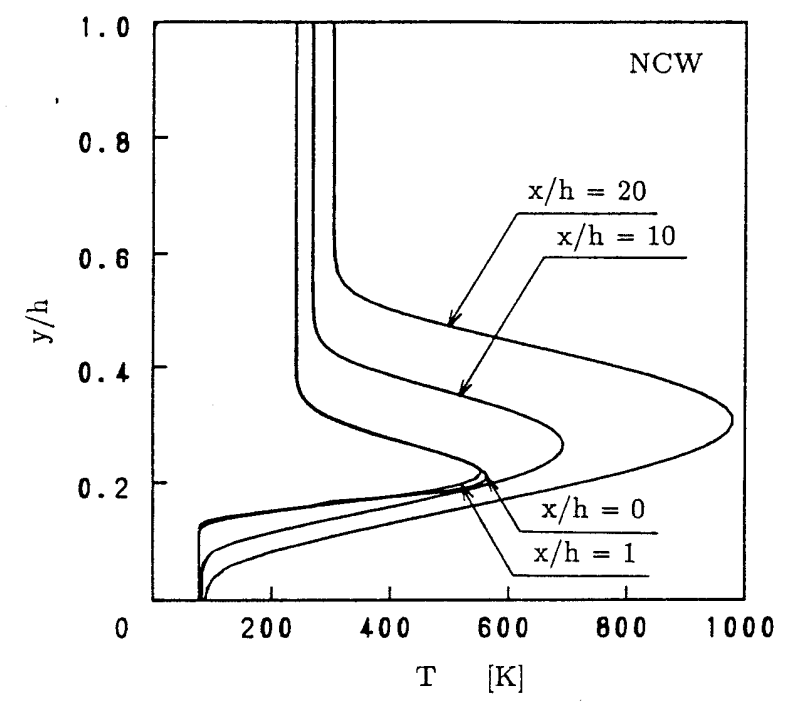

Fig. 7 Distributions of the temperature obtained assuming the noncatalytic wall

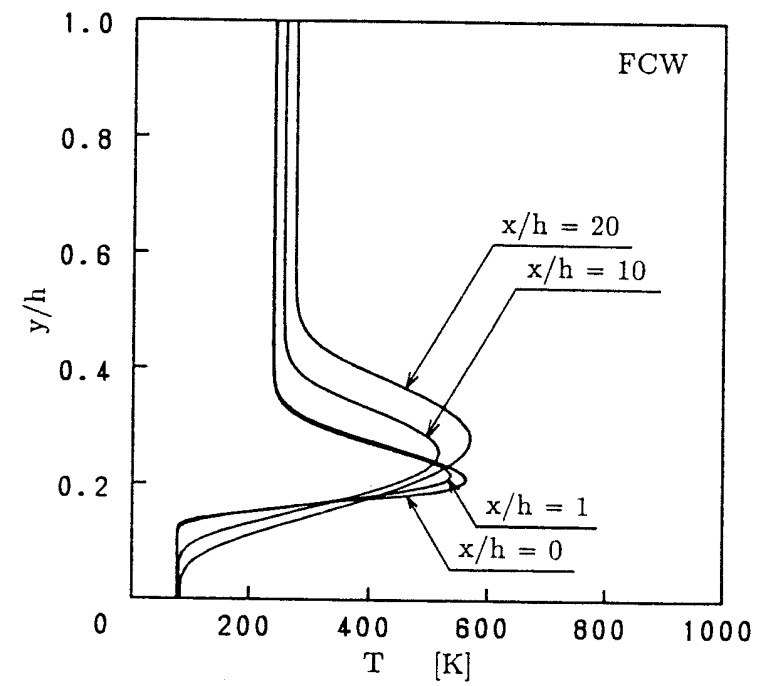

Fig. 8 Distributions of the temperature obtained assuming the fully catalytic wall 
the wake issuing from the nozzle trailing edge increases and the wake region disappears completely in the distributions at $x / h=10$. The shear layer develops in the downstream direction. However, the growth is very gradual as was observed from Fig. 2. The regions near the nozzle centerlines are still inviscid even at $x / h=20$. Comparing the distributions shown in Figs. 9 and 10, it is seen that the assumption of the noncatalytic wall condition leads to somewhat faster growth of the shear layer than that of a fully catalytic wall; the reason is that the assumption of the noncatalytic wall condition results in the higher temperature rise and, thereby, the higher viscosity.

The distributions of the small signal gain coefficients $G$ and the temperature at $x / h=10$ and 20 obtained by assuming the noncatalytic wall condition

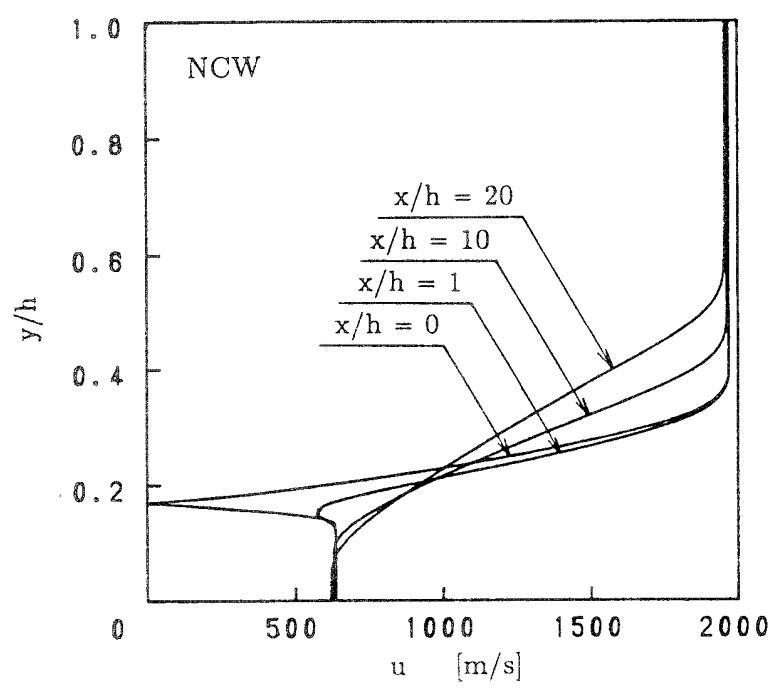

Fig. 9 Velocity distributions obtained assuming the noncatalytic wall

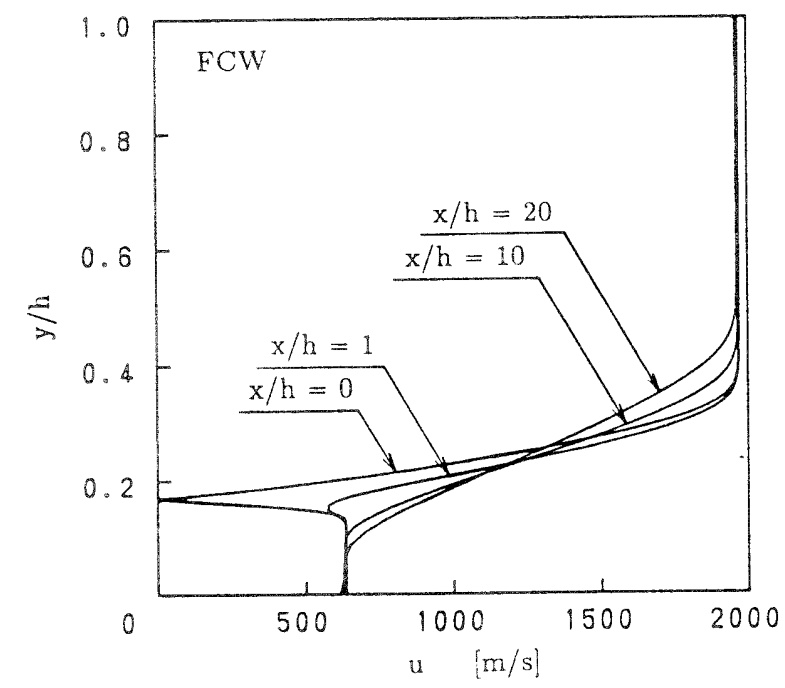

Fig. 10 Velocity distributions obtained assuming the fully catalytic wall are shown in Figs. 11 and 12, respectively. It is seen from the distributions at $x / h=10$ that the maximum gains of nearly $1 \mathrm{~m}^{-1}$ can be attained in the mixing layer. However, the active region where the gains are positive is only about one third of the flow width. Figure 12 shows that the maximum gains at $x / h=20$ are already decaying in the downstream direction, although the mixing layer is still a small portion of the total flow field. These results show that much smaller nozzles than those in the previous experiment are desirable in order to attain a higher average gain. The gains of all branches shown in Fig. 12 are reduced

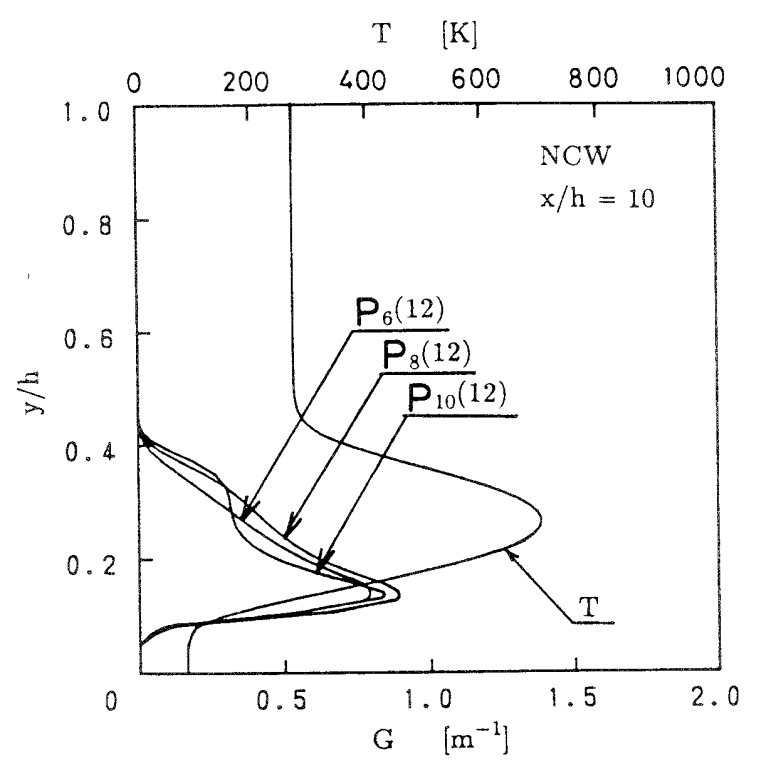

Fig. 11 Distributions of the small signal gain coefficients and the temperature at $x / h=10$ obtained assum. ing the noncatalytic wall

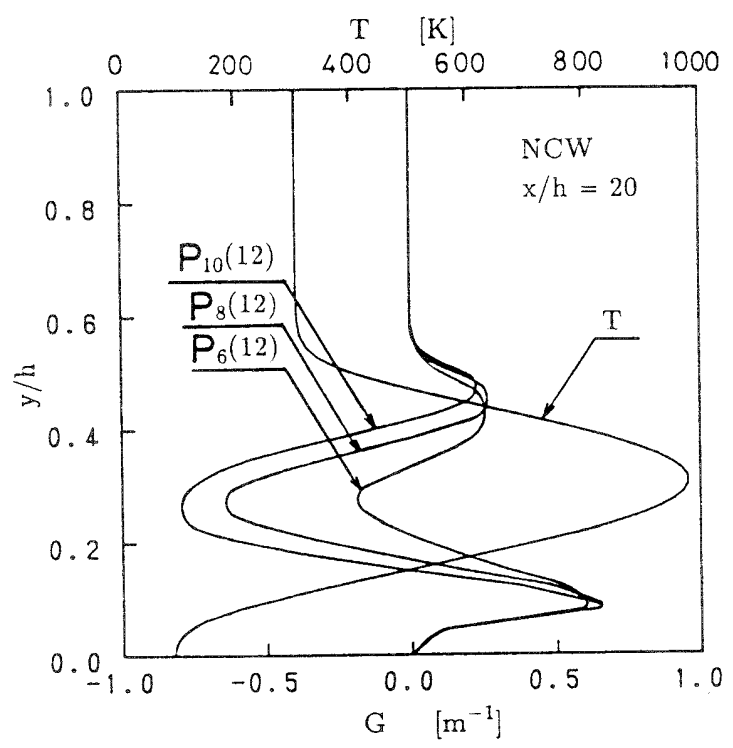

Fig. 12 Distributions of the small signal gain coefficients and the temperature at $x / h=20$ obtained assuming the noncatalytic wall 
severely and are negative where the temperature is very high since vibrational relaxation is promoted. It is pointed out that a rise in temperature does not lead to rapid formation of the vibrationally excited $\mathrm{CO}$ molecules, since the dependence of the rate constants for reactions ( 1 ) and ( 2 ) on the temperature is very weak. Conversely, the vibrational relaxation is strongly promoted as the temperature is raised. Moreover, the small signal gain coefficients are reduced by the temperature rise, since the spectral line width spreads. Therefore, the temperature rise is significantly detrimental to the laser performance.

The dependence of the small signal gain coefficient of the $P_{8}(12)$ branch on $x$ is shown in Fig. 13 where $\bar{G}$ is the small signal gain coefficient averaged in the $y$ direction. It is seen that the peaks of the averaged gains obtained by the calculation and the experiment agree fairly well with each other. However, the locations of the peaks and widths of the active region are considerably different. The peak value of the averaged gain obtained by assuming the noncatalytic wall condition is only slightly higher than that obtained assuming the fully catalytic wall condition, though the mole fraction of $\mathrm{CO}$ depends strongly on the magnitude of the wall catalysis as shown in Figs. 5 and 6 . However, the active region obtained by assuming the fully catalytic wall condition is consider. ably wider than that obtained by assuming the noncatalytic wall condition. These results clearly demonstrate that the effect of the temperature rise in the mixing layer is significantly detrimental to the laser performance. These results also show clearly that the existence of the nozzle boundary layers and the wall catalysis play very important roles in determining the

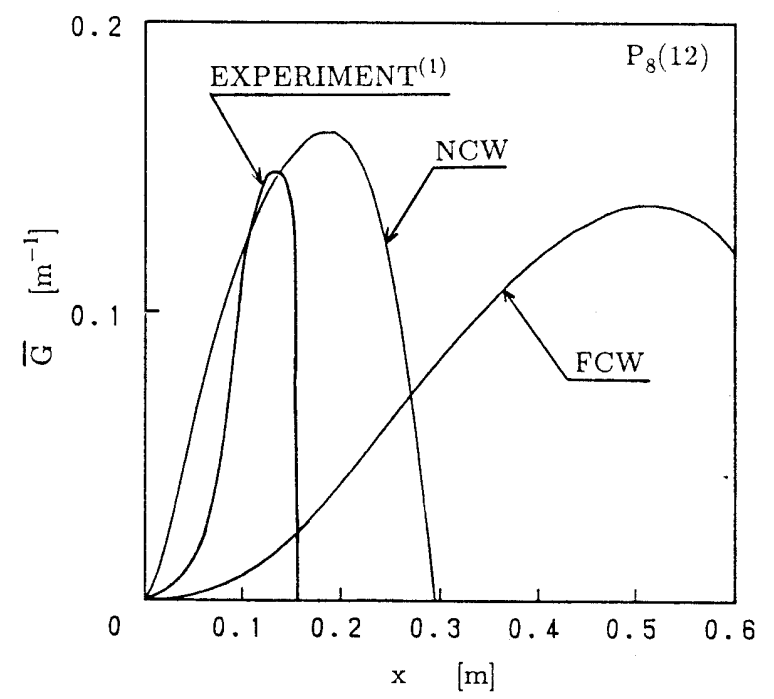

Fig. 13 Distributions of $\bar{G}$ along the flow obtained assuming the presence of the nozzle boundary layers laser performance.

The effects of the cavity boundary layers are examined in what follows. Figures 14 and 15 shows the distributions of the temperature and the mole fractions of the species obtained by assuming the fully catalytic wall condition and the existence of the cavity boundary layers. The existence of boundary layers causes the convergence of the effective cross sectional area of the cavity. Therefore, the temperature of the cavity flow is raised steeply in the downstream direction as can be seen from Figs. 8 and 14. Although the rise in the temperature does not lead to the fast rate constants for reactions (1) and (2), the mole fraction of $\mathrm{CO}$ in the mixing region is increased slightly due to the presence of the cavity boundary layers as shown in Figs. 6 and 15 ; the reason is that the reac.

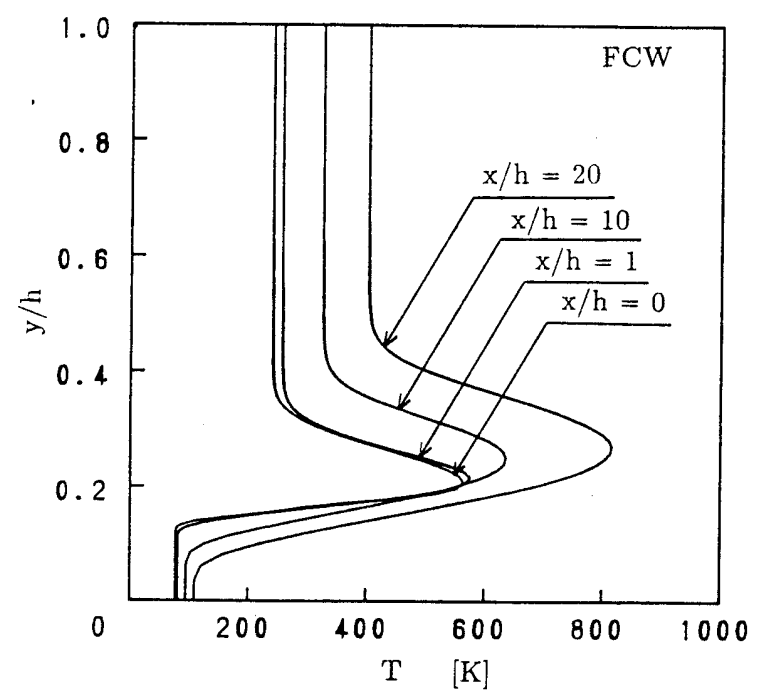

Fig. 14 Distributions of the temperature obtained assuming the fully catalytic wall

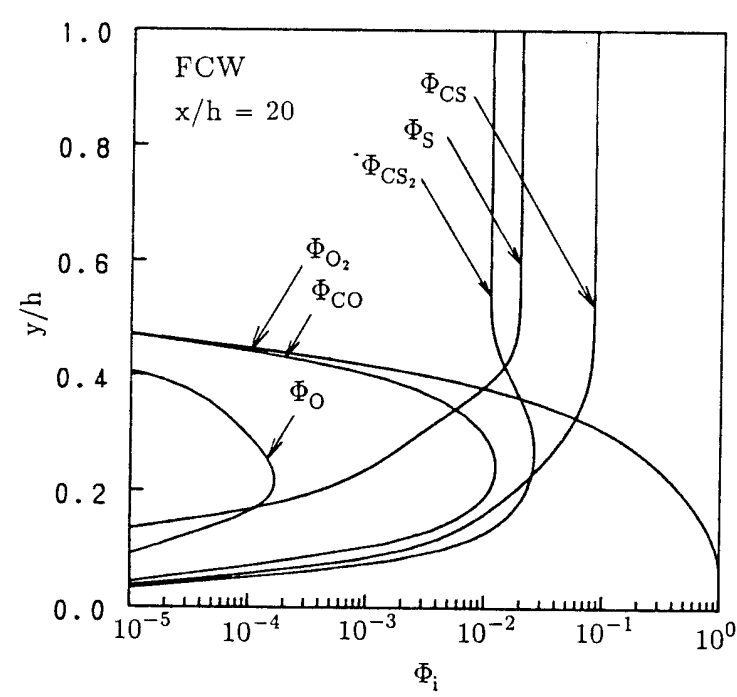

Fig. 15 Distributions of the mole fractions of species obtained assuming the fully catalytic wall 


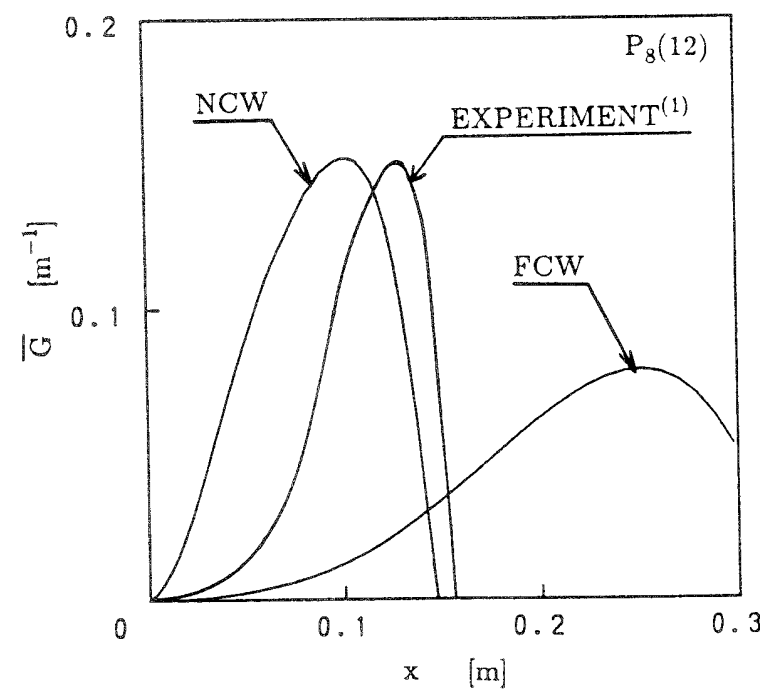

Fig. 16 Distributions of $\bar{G}$ along the flow obtained assuming the presence of the nozzle boundary layers and the cavity boundary layers

tions are promoted due to the increase in molecular density.

The dependence of the averaged small signal gain coefficients on $x$ obtained assuming the presence of the nozzle boundary layers and the cavity boundary layers is shown in Fig. 16 and compared with the experimental result. It is clearly seen that the presence of the cavity boundary layers results in the lower peak of the averaged gain and narrower active region. The numerical result obtained by assuming the noncatalytic wall condition agrees considerably well with the experiment. However, the present results are insufficient to suggest that the nozzle wall was nearly noncatalytic, since the present estimation of the threedimensional cavity boundary layer is very rough. Moreover, the effects of the oblique shock waves observed in Fig. 2 are not included in the present model. The presence of the oblique shock waves leads to the higher temperature and higher density as like that of the cavity boundary layers. Therefore, it is imperative to model accurately the three-dimensional cavity boundary layers and the oblique shock waves in order to predict the gain characteristics more precisely. However, the present study distinctly demonstrates that the presence of the nozzle boundary layers and the cavity boundary layers plays decisive roles in determining laser performance. It must also be pointed out that the gain characteristics greatly depend on the magnitude of the wall catalysis.

\section{Conclusions}

The present calculations show that the develop- ment of the mixing layer in the downstream direction is very gradual as was demonstrated by the visual observation of the flow field. The small signal gain coefficients begin to decrease at a location where the mixing layer is still a small portion of the total flow width. Therefore, much smaller nozzles than those of the previous experiment are desirable in order to attain a higher average gain. The predicted distributions of the averaged gain along the flow agree fairly well with the previous experiment. It is clearly demonstrated that the presence of the boundary layers and the magnitude of the wall catalysis play decisive roles in determining laser performance.

This work has been partly supported by a Grantin-Aid for Scientific Research from the Ministry of Education, Science, and Culture of Japan.

\section{References}

(1) Masuda, W., Ikarashi, M. and Shiraishi, K., Measurements of Small Signal Gain Coefficients of a Supersonic Flow CO Chemical Laser, Trans. Jpn. Soc. Mech. Eng., (in Japanese), Vol. 55, No. 512 (1989), p. 1097, also to be published in JSME Int. J.

(2) Masuda, W. and Tohyama, M., Numerical Simulation of a Supersonic Flow CO Chemical Laser Using a Leaky Stream Tube Approach, Trans. Jpn. Soc. Aero. Space Sci., Vol. 30, No. 90 (1988), p. 201.

(3) Masuda, W. and Hiraide, H., Investigation on the Theoretical Modeling of a Supersonic Flow CO Chemical Laser, Trans. Jpn. Soc. Mech. Eng., (in Japanese), Vol. 55, No. 512 (1989), p. 1101, also to be published in JSME Int. J.

(4) Masuda, W., Ikarashi, M., Shiraishi, K. and Hiraide, H., Studies on Supersonic Flow CO Chemical Laser, Proc. 7th Int. GCL Symp, to be published.

(5) Richardson, R. J., CS-O, Flame Reaction Chemistry, J. Phys. Chem., Vol. 79, No. 12 (1975), p. 1153.

(6) Behrens, W. and Ko., D. R. S., Experimental Stability Studies in Wakes of Two-Dimensional Slender Bodies at Hypersonic Speed, AIAA J., Vol. 9. No. 5 (1971), p. 851.

(7) Matsuno, K., A Second-Order Accurate Procedure for Solving the Boundary Layer Equations Based on the Predictor-Corrector Form of the Crank-Nicolson Scheme, NAL TR-655 T(1981).

(8) Russel, D. A., Fluid Mechanics of High Power Grid Nozzle Lasers, AIAA Paper, No. 74223(1974).

(9) Masuda, W., Ogawahara, N. and Tohyama, M., Gain Characteristics of a Supersonic Flow CO Chemical Laser, Trans. Jpn. Soc. Aero. Space Sci., Vol. 30, No. 87 (1987), p. 1.

(10) Miner, E. W. and Lewis, C. H., Hypersonic Ionizing Air Viscous Shock-Layer Flows over Nonanalytic Blunt Bodies, NASA CR-2550 (1975). 